\title{
Análisis de la demanda en Salud Mental en el Principado de Asturias (II): Expectativas, preferencias e información de los usuarios.
}

Mental Health demand analysis in the Principality of Asturias (II): User expectations, preferences and information.

Fátima Valencia Agudo a , Juan García Haro o ${ }^{\text {, }}$ María Eugenia de la Viuda Suárez ${ }^{\mathrm{c}}$, Ana Pérez

Zabalza d, Alberto Gimeno Peón e.

${ }^{a, c, d y e}$ Psicólogo Interno Residente. ${ }^{b}$ Psicólogo Clínico. ${ }^{a, b, c, d y e}$ Servicios de Salud Mental del Principado de Asturias, España.

Correspondencia: Fátima Valencia Agudo (fatima-valencia@hotmail.es)

Recibido: 12/08/2014; aceptado con modificaciones: 07/03/2015

RESUMEN: Introducción. Las expectativas y preferencias del paciente son una cuestión fundamental a tener en cuenta en Salud Mental. Por una parte, el derecho a decidir entre las diferentes opciones terapéuticas se recoge en la Ley de Autonomía del Paciente. Por otro lado, múltiples investigaciones han mostrado la influencia de las expectativas del usuario acerca del tratamiento, y por tanto la importancia de tenerlas en consideración. En este sentido, la información proporcionada por parte del derivante cobra especial relevancia. Método. La muestra se toma de usuarios derivados a Salud Mental que cumplen los criterios de inclusión y aceptan participar en el estudio $(n=121)$. Mediante una entrevista telefónica se recoge información de variables sociodemográficas, relacionadas con la derivación, con las preferencias del paciente y finalmente con sus expectativas.

Resultados y conclusiones. Respecto a las variables de la derivación, encontramos que el derivante proporcionó escasa información al paciente acerca de su problema y de los diferentes tipos de tratamiento. En las variables asociadas a las preferencias destaca el desconocimiento de los usuarios acerca de las labores del psicólogo clínico y del psiquiatra, así como de las diferentes implicaciones de la psicoterapia y del tratamiento psicofarmacológico. En cuanto a las expectativas, observamos de nuevo falta de información pero además un nivel importante de confianza en la intervención y una puntuación alta en locus de control interno. Parece fundamental una mayor consideración de este tipo de variables para la mejora del proceso de intervención posterior.

PALABRAS CLAVE: Análisis de la demanda, Ley de Autonomía del Paciente, Atención Primaria, Salud Mental, preferencias del paciente, expectativas del paciente.
ABSTRACT: Background. Patient expectations and preferences are fundamental aspects to take into account in Mental Health. On the one hand, the right to decide between different treatment options is included in the Law of Patient Autonomy. On the other hand, research has shown the influence of patient treatment expectations and therefore the importance of taking them into consideration. In this regard, the information provided by the professional referring the patient gains special relevance.

Method. The sample is taken from the patients referred to Mental Health Services who meet the inclusion criteria and accept to participate in the study $(n=121)$. We collect sociodemographic variables as well as variables related to the referral process and to preferences and expectations by means of a telephone survey.

Results and conclusions. Regarding the referral process, we found that the professional who referred the patient provided little information about his problem and the different treatment options available. With respect to preferences, we point out the lack of knowledge about differences between the tasks of a clinical psychologist and a psychiatrist, as well as the different implications of psychotherapy and psychopharmacologic treatment. Regarding expectations, we observe again lack of information but also an important level of confidence in the intervention and a high internal locus of control. It seems important to take these aspects into further consideration in order to improve the intervention process.

KEY WORDS: Demand analysis, Law of Patient Autonomy, Primary Health Care, Mental Health, patient preferences, patient expectations. 
ORIGINALES Y REVISIONES

\section{Introducción}

Este artículo es parte de un proyecto que pretende conocer las características de la demanda y el tipo de respuesta asistencial ofrecida en los Servicios de Salud Mental del Principado de Asturias. En este trabajo nos centraremos en cuestiones que consideramos fundamentales en nuestro ámbito de trabajo: la capacidad de decisión del paciente acerca de las opciones de tratamiento y las expectativas que este tiene acerca de la ayuda que recibirá.

A la hora de tratar estas cuestiones, es necesario empezar hablando de la Ley de Autonomía del Paciente (Ley 41/2002, de 14 de Noviembre, básica reguladora de la autonomía del paciente y de derechos y obligaciones en materia de información y documentación clínica). Los aspectos fundamentales recogidos en dicha ley son los siguientes: la confidencialidad de los datos personales, la necesidad de consentimiento previo por parte del paciente antes de llevar a cabo cualquier actuación sanitaria y el derecho a decidir libremente entre las alternativas disponibles de tratamiento, después de haber sido debidamente informado. Este último punto es el eje central de este trabajo; implica que el facultativo responsable del caso debe informar al paciente acerca de las diferentes opciones clínicas, así como de sus beneficios y contraindicaciones, para que una vez en posesión de los datos la persona pueda decidir qué tipo de tratamiento considera más adecuado.

En Salud Mental podemos distinguir básicamente tres tipos de intervenciones generales: psicoterapia, tratamiento psicofarmacológico y tratamiento combinado. La importancia del derecho del paciente a elegir un tratamiento no es una mera cuestión legalista, sino que tiene implicaciones para la evolución y el resultado clínico. Por tanto también para la evaluación de costes asociados a las intervenciones sanitarias. Las elecciones y preferencias de los usuarios no sólo son cuestiones subjetivas sino que tienen consecuencias prácticas en el funcionamiento del proceso terapéutico y, por tanto, en los resultados obtenidos. Basta con pensar en un persona que sufre ataques de ansiedad y prefiere benzodiazepinas en lugar de aplicar estrategias psicológicas. De nada servirá proponerle, por ejemplo, la exposición a sensaciones corporales de malestar si no considera que dicha intervención le será de utilidad. En primer lugar, lo más probable es que no lleve a cabo la tarea indicada y en segundo lugar las nulas expectativas de mejoría contrarrestarán la eficacia que pudiera tener la técnica. Lo mismo puede suceder en el caso contrario: si un usuario no considera que su problema se vaya a solucionar con tratamiento farmacológico, entonces cabe esperar que no tome dicho tratamiento o lo haga de forma irregular, o que lo tome pero que las expectativas de no mejoría pesen más que el efecto específico del fármaco. Recordemos el estudio clásico de Lambert y Anderson (1) en el que se encontró que el peso de las expectativas en el resultado de la terapia era del $15 \%$, igual al de la aplicación de determinadas técnicas. Además, las expectativas tienen mucho que 
ORIGINALES Y REVISIONES

ver con los factores comunes en psicoterapia (2) y con el denominado efecto placebo, cuya influencia en el resultado clínico es ampliamente conocida (3), llegando incluso a encontrarse una mayor eficacia del placebo que de otro tipo de tratamientos (4-6). Dentro del ámbito de la psicoterapia diversos autores han planteado la importancia de las expectativas del paciente sobre el resultado de la intervención (7-8). La investigación ha determinado de forma empírica dos tipos de expectativas: expectativas de resultado, creencias acerca de la eficacia que tendrá el tratamiento, y expectativas de la terapia, relacionadas con cómo será el proceso de intervención (p.e. cómo se comportará el terapeuta, cómo se sentirá el paciente o la duración de la misma) (9).

Respecto a las variables relacionadas a las expectativas, existen evidencias empíricas de que los usuarios esperan recibir pocas sesiones (10-11). Se podría cuestionar que "pocas sesiones" sean suficientes para la resolución de problemas psicológicos o para la mejoría clínica, pero no es así. En relación a los tratamientos psicológicos, diversos estudios empíricos indican que las psicoterapias breves (entre 6 y 12 sesiones) de diferentes marcos teóricos son realmente efectivas. Esto no sólo se verifica en contextos controlados de investigación, sino también en la práctica real de los servicios públicos. Así, en un estudio que compara la efectividad de la psicoterapia breve con el tratamiento habitual, realizado en los servicios públicos de Salud Mental de Asturias (12-13), aplicada al conjunto de los trastornos mentales comunes, se encontró que la psicoterapia breve resultaba más efectiva que el tratamiento habitual en numerosas variables de resultado. Por otro lado, diversos estudios indican que las psicoterapias breves son en general tan eficaces como las de mayor duración (14). También en relación a las expectativas, existen estudios que señalan que los usuarios esperan ser atendidos por psicólogos más que por psiquiatras. Así, en un estudio realizado en el Servicio de Salud de Vizcaya (15) se encontró que los usuarios esperaban ser atendidos por psicólogos en el 48,3\% de los casos, mientras que el $25,9 \%$ esperaban ser atendidos por psiquiatras.

Respecto de las variables relacionadas a las preferencias, existe evidencia acerca de que los usuarios prefieren la terapia psicológica antes que la opción farmacológica (16-17).

Estos datos han dado lugar a diversas propuestas para ajustar las psicoterapias a las expectativas y preferencias del paciente (18-19). Asimismo, también se han propuesto estrategias para intervenir en las expectativas que los usuarios tienen, con el objetivo de mejorar el resultado clínico posterior (9).

Estos planteamientos enfatizan la importancia de tener en cuenta las expectativas y preferencias del usuario en torno a diferentes tipos de psicoterapia, que aunque presenten diferentes objetivos y diferentes medios para conseguirlos, tienen en común múltiples factores (p.e. el establecimiento de una relación terapéutica o fomentar un afrontamiento alternativo de las dificultades). Por tanto, ajustar la intervención al paciente cuando estamos hablando de tratamientos tan diferentes como el psicoterapéutico y el psicofarmacológico cobrará mayor importancia si cabe. 
Además de las preferencias del usuario también hay que tener en consideración la valoración del clínico acerca de la mejor intervención en función del paciente, especialmente si tenemos en cuenta que en algunos casos (por ejemplo, anorexia o psicosis) el individuo ni siquiera reconoce la necesidad de llevar a cabo un tratamiento. Otra posibilidad es que personas que no presentan ninguna alteración psicopatológica, sino que únicamente están atravesando por una circunstancia estresante, consideren que han de someterse a una intervención especializada en Salud Mental. Para manejar estas situaciones es fundamental conocer las expectativas de los usuarios, para poder en ocasiones ajustarnos a ellas y otras veces aclararlas o modificarlas, tratando de equilibrar el respeto por los derechos del paciente con el conocimiento que tenemos del funcionamiento psicológico y psicopatológico humano. En algunos casos cobrará especial importancia el trabajo clínico relacionado con la motivación (20) y en la mayoría de las ocasiones será fundamental dar al paciente la información acerca de los resultados esperables según cada línea de actuación.

La intervención del derivante tiene una influencia fundamental en las expectativas de los usuarios que solicitan consulta en Salud Mental. Las estrategias para modificar las expectativas de los pacientes tienen que ver en gran medida con la información (9). De ahí la importancia de la actuación del Médico de Atención Primaria en el proceso de derivación, ya que puede ser una pieza clave a la hora de fomentar la decisión informada de los pacientes de acuerdo a la Ley de Autonomía. Respecto a las variables relacionadas con la derivación, existe un estudio realizado en centros de Atención Primaria de Córdoba (21) donde se pregunta a los médicos de familia acerca de la información dada a los usuarios cuando se les deriva a Salud Mental. Se encuentra que casi el $80 \%$ de los MAP reconocían no utilizar el consentimiento informado sobre el tratamiento, ni explicar que existen alternativas para solucionar los problemas como el tratamiento psicológico (como opción terapéutica previa antes de prescribir medicación), el tratamiento combinado u otras. En este mismo estudio, los médicos de familia referían que el $39 \%$ de sus pacientes tratados con fármacos podrían resolver sus problemas psicológicos sin hacer uso de ellos.

Los objetivos de este trabajo son los siguientes: a) explorar qué tipo de información han recibido por parte de sus derivantes los usuarios que son derivados por primera vez a los Servicios de Salud Mental, b) conocer sus preferencias en cuanto a tipo de tratamiento así como sus expectativas e información en torno a variables relacionadas con la intervención, c) examinar si existe coincidencia entre las preferencias de tratamiento y el profesional asignado en atención especializada. 
ORIGINALES Y REVISIONES

\section{Método}

\section{Muestra}

La muestra se toma de aquellos usuarios derivados a tres Centros de Salud Mental (CSM en adelante) del Servicio de Salud del Principado de Asturias entre los meses de febrero y mayo de 2012. Los criterios de exclusión fueron tener menos de 18 años, no presentar el volante de derivación, haber sido atendido previamente en el centro (ser un retorno) y tener antecedentes en Salud Mental Infanto-juvenil. Por otra parte no se incluyen en el estudio pacientes con los que fue imposible contactar, pacientes con los que fue imposible comunicarse por motivos clínicos, aquellos casos en los que la persona que solicitó la derivación no coincidía con el paciente y casos en los que el usuario rechazaba la participación en el estudio. En total, cumplen criterios de inclusión y aceptan participar en la investigación el 31,8\% de las personas derivadas $(\mathrm{n}=121)$.

\section{Procedimiento}

Se trata de un estudio descriptivo observacional que se lleva a cabo en tres CSM. En cada centro había un responsable de la recogida de la información, que examinaba las derivaciones. En caso de que el paciente reuniese criterios de inclusión se contactaba con él vía telefónica. Se le explicaban los objetivos del estudio y si aceptaba participar se aplicaba un consentimiento informado y se realizaba una entrevista telefónica.

Una vez realizadas todas las llamadas se categorizaron y analizaron las respuestas mediante el paquete estadístico SPSS v. 17. Se llevaron a cabo pruebas de estadística descriptiva a fin de conocer la distribución de las variables y pruebas de Chi Cuadrado $\left(\chi^{2}\right)$ para comprobar la relación existente entre variables nominales.

El estudio cuenta con la aprobación de las tres direcciones de Área de Gestión Clínica y del Comité Ético de Investigación Clínica Regional del Principado de Asturias.

Variables

Variables relacionadas con la derivación

- Informe de la derivación. Se refiere a si el derivante le explicó el motivo de la derivación y en tal caso qué le dijo.

- Informe del problema. Se pregunta si el derivante le dio algún tipo de explicación acerca de lo que le ocurría.

- Informe de los tratamientos. Se trata de comprobar si el derivante informó acerca de las alternativas de tratamiento y de las ventajas e inconvenientes de cada una.

En las variables anteriores las categorías se realizaron a posteriori en función de las respuestas obtenidas.

- Conformidad con la derivación. Se daba a escoger entre las siguientes opciones de respuesta: sí, no, sí con reservas. 
- Satisfacción con el proceso de derivación. Valorada en una escala de 1 a 10.

\section{Variables relacionadas con las preferencias del usuario}

- Preferencia de terapeuta. Se da a escoger entre las siguientes alternativas: psicólogo clínico, psiquiatra, no sabe/no contesta.

- Preferencia de tratamiento. Las opciones de respuesta son las siguientes: psicoterapia, tratamiento farmacológico, tratamiento combinado, no sabe/ no contesta.

- Frecuencia de consulta deseada. Las opciones de frecuencia de consulta son estas: semanal, quincenal, mensual, otra, no sabe/no contesta.

\section{Variables relacionadas con las expectativas del usuario}

- Frecuencia de consulta esperada. Las opciones de respuesta son las mismas que en el caso de la variable frecuencia de consulta deseada.

- Número de consultas necesarias. Número de sesiones que cree que serán necesarias para la resolución del problema por el que acude a consulta.

- Confianza en la ayuda que recibirá en Salud Mental. Valorada en una escala de 1 a 10.

- Locus de control interno. Se pidió a los participantes que valorasen en una escala de 1 a 5 (siendo 1 "nada de acuerdo" y 5 "muy de acuerdo") la siguiente afirmación: "La solución de mi problema tendrá mucho que ver con el empeño que yo ponga en resolverlo".

\section{Resultados}

En la tabla 1 se presentan las características sociodemográficas de la muestra.

Con respecto a las variables de estudio, encontramos los siguientes resultados: Variables relacionadas con la demanda

- Informe de derivación. Al preguntar a los participantes si sabían por qué su médico les había derivado a Salud Mental, el 29,2\% (n=35) respondió que lo habían pedido ellos mismos o alguien de su entorno, un $21,7 \%(\mathrm{n}=14)$ que tenían un problema psicológico o de salud mental, el 11,7\% $(\mathrm{n}=14)$ contestó que fue debido a sus circunstancias psicosociales, el $10,8 \%(\mathrm{n}=13)$ que precisaban atención especializada y un $6,7 \%(\mathrm{n}=8)$ no conocían el motivo por el que fueron derivados. Un 7,5\% $(\mathrm{n}=9)$ aportan información que no corresponde con el motivo de derivación y un 7,14\% $(\mathrm{n}=8)$ recibió otro tipo de explicación.

- Informe del problema. El 59,5\% (n=72) de los participantes refiere no haber recibido ninguna explicación acerca del origen o de las características del problema, el $10,7 \%(\mathrm{n}=13)$ fue informado de que su problema tenía que ver con circunstancias psicosociales, el $8,3 \%(\mathrm{n}=10)$ de que tenía un problema 
de salud mental y finalmente al $4,1 \%(\mathrm{n}=5)$ se les explicó que sus síntomas somáticos podían tener un origen o influencia psicógena. Un 9,1\% $(\mathrm{n}=11)$ no especifica información relacionada con un informe del problema y el 8,3\% $(\mathrm{n}=10)$ de las respuestas son otras explicaciones distintas acerca del problema.

- Informe de los tratamientos. Un 78\% ( $\mathrm{n}=92)$ de los usuarios refiere que el derivante no le explicó las alternativas de tratamiento, un $4,2 \%(\mathrm{n}=5)$ responde que su médico le habló de las opciones terapéuticas (fármacos - psicoterapia) y en un 7,6\% $(\mathrm{n}=9)$ de los casos le dio además su opinión profesional. Un 4,2\% $(n=5)$ solo fue informado acerca del tratamiento farmacológico que le pautó su médico. Un 5,1\% $(\mathrm{n}=6)$ aporta información que no corresponde con una explicación de las alternativas de tratamiento y un caso $(0,8 \%)$ recibió otro tipo de informe diferente a los anteriores.

- Conformidad con la derivación. Un 87,5\% $(n=103)$ se muestra de acuerdo con la derivación, un $10,2 \%(\mathrm{n}=12)$ está conforme pero tiene reservas y un $1,7 \%(\mathrm{n}=2)$ no está de acuerdo con la derivación. Un sujeto $(0,8 \%)$ no sabe/ no contesta.

- Satisfacción con la derivación. La mediana fue de 9.

Tabla 1.

Características sociodemográficas de la muestra.

\begin{tabular}{|l|c|}
\hline Variable sociodemográfica & Valor \\
\hline Edad & 41,49 \\
Media & 13,73 \\
\hline Desviación típica & \\
\hline Sexo & $57,02 \%(\mathrm{n}=69)$ \\
Mujeres & $42,97 \%(\mathrm{n}=52)$ \\
\hline Hombres & \\
\hline Convivencia & $25,6 \%(\mathrm{n}=31)$ \\
Familia origen & $51,2 \%(\mathrm{n}=62)$ \\
Familia propia & $18,2 \%(\mathrm{n}=22)$ \\
Solo & $4,1 \%(\mathrm{n}=5)$ \\
Con otras personas & $0,8 \%(\mathrm{n}=1)$ \\
Institución o piso protegido & \\
\hline Estado civil & $54,2 \%(\mathrm{n}=65)$ \\
Casado o en pareja & $28,3 \%(\mathrm{n}=34)$ \\
Soltero & $11,7 \%(\mathrm{n}=14)$ \\
Separado o divorciado & $5,7 \%(\mathrm{n}=7)$ \\
Viudo & \\
\hline Situación laboral & $31 \%(\mathrm{n}=36)$ \\
Activo & $24,13 \%(\mathrm{n}=28)$ \\
Parado & $7,8 \%(\mathrm{n}=9)$ \\
Jubilado o pensionista & $8,6 \%(\mathrm{n}=10)$ \\
Estudiante & $7,8 \%(\mathrm{n}=9)$ \\
Labores del hogar & $20,7 \%(\mathrm{n}=24)$ \\
Incapacidad laboral temporal & \\
\hline Medio & $79,8 \%(\mathrm{n}=95)$ \\
Urbano & $20,2 \%(\mathrm{n}=24)$ \\
\hline Rural & \\
\hline & \\
\hline
\end{tabular}


Variables relacionadas con las preferencias del usuario

- Preferencia de terapeuta. Un 19\% $(\mathrm{n}=23)$ de los usuarios prefieren psicólogo clínico mientras que un $11,6 \%(\mathrm{n}=14)$ prefieren psiquiatra. Por otra parte, un $69,4 \%(\mathrm{n}=84)$ no sabe/no contesta.

- Preferencia de tratamiento. El 35,5\% $(n=43)$ de la muestra dice preferir psicoterapia, el 14\% $(n=17)$ tratamiento combinado y el 1,7\% $(n=2)$ tratamiento farmacológico. Finalmente el $48,8 \%(\mathrm{n}=59)$ no sabe/no contesta.

- Frecuencia de consulta deseada. El 13,3\% (n=16) de la muestra responde que desearía que la consulta tuviese una frecuencia quincenal, el $9,2 \%$ $(\mathrm{n}=11)$ mensual, el 5,8\% $(\mathrm{n}=7)$ semanal y el $6,6 \%(\mathrm{n}=8)$ otro tipo de periodicidad. Un $54,2 \%(\mathrm{n}=65)$ no sabe/no contesta y un $10,8 \%(\mathrm{n}=13)$ aporta una respuesta que no corresponde con la pregunta realizada.

\section{Variables relacionadas con las expectativas del usuario}

- Frecuencia de consulta esperada. Un 7,5\% $(n=9)$ responde que espera que la consulta sea mensual, un $6,7 \%(\mathrm{n}=8)$ quincenal, un $2,5 \%(\mathrm{n}=3)$ semanal y un $4,1 \%(\mathrm{n}=5)$ tiene otro tipo de expectativa. Un 72,5\% $(\mathrm{n}=87)$ no sabe/no contesta y un $6,7 \%(\mathrm{n}=8)$ no especifica la información por la que es preguntado.

- Número de consultas necesarias. Un 11,8\% $(\mathrm{n}=14)$ considera que necesitará entre 1 y 5 consultas, un $10,9 \%(n=13)$ entre 6 y 10 consultas, un $3,4 \%(n=4)$ entre 11 y 15 consultas y un $2,5 \%(\mathrm{n}=3)$ más de 16 consultas. Un $67,2 \%$ $(\mathrm{n}=80)$ no sabe/no contesta y un $4,2 \%(\mathrm{n}=5)$ no especifica la información.

- Confianza que tiene en la ayuda. La mediana fue de 8.

- Locus de control interno. La mediana fue 5, correspondiente con la máxima puntuación de la escala.

\section{Pruebas de Chi Cuadrado ( $\left.\chi^{2}\right)$}

Para llevar a cabo dicho análisis se recogió información acerca del terapeuta asignado al paciente en el CSM.

- Con respecto a la coincidencia entre el profesional preferido por el paciente y el profesional asignado, parece que sí existe dicha asociación $\left(\chi^{2}=6,684, p<0,05\right)$.

Tabla 2.

Relación entre el profesional preferido y el profesional asignado

\begin{tabular}{|l|c|c|c|c|}
\hline Profesional preferido & & $\begin{array}{c}\text { Asignación Psicólogo } \\
\text { Clínico }\end{array}$ & $\begin{array}{c}\text { Asignación } \\
\text { Psiquiatra }\end{array}$ & Total \\
\hline \multirow{2}{*}{ Psicólogo Clínico } & $\mathrm{n}$ & 15 & 8 & 23 \\
& $\%$ & $65,21 \%$ & $34,78 \%$ & $100 \%$ \\
Psiquiatra & $\mathrm{n}$ & 3 & 11 & 14 \\
& $\%$ & $21,42 \%$ & $78,57 \%$ & $100 \%$ \\
No sabe/no contesta & $\mathrm{n}$ & 40 & 43 & 83 \\
& $\%$ & $48,19 \%$ & $51,8 \%$ & $100 \%$ \\
\hline Total & & 58 & 62 & 120 \\
\hline
\end{tabular}

$\chi^{2}=6,684 ; \mathrm{p}<0,05$ 
En cuanto a la relación entre el tipo de tratamiento preferido y el profesional asignado (asumiendo que cada uno realiza un tipo de intervención), también parece haber coincidencia $\left(\chi^{2}=32,633, p<0,05\right)$. Cabe mencionar que en nuestra Comunidad Autónoma la psicoterapia es llevada a cabo mayoritariamente por parte de psicólogos clínicos.

Tabla 3.

Relación entre preferencia de tratamiento y profesional asignado.

\begin{tabular}{|l|c|c|c|c|}
\hline Preferencia de tratamiento & & $\begin{array}{c}\text { Asignación Psicólogo } \\
\text { Clínico }\end{array}$ & $\begin{array}{c}\text { Asignación } \\
\text { Psiquiatra }\end{array}$ & Total \\
\hline Psicoterapia & $\mathrm{n}$ & 35 & 7 & 42 \\
& $\%$ & $83,33 \%$ & $16,66 \%$ & $100 \%$ \\
Tratamiento farmacológico & $\mathrm{n}$ & 0 & 2 & 2 \\
& $\%$ & - & $100 \%$ & $100 \%$ \\
Combinado & $\mathrm{n}$ & 6 & $64,7 \%$ & 17 \\
& $\%$ & $35,29 \%$ & 42 & $100 \%$ \\
No sabe/no contesta & $\mathrm{n}$ & 17 & $71,18 \%$ & 59 \\
\hline Total & $\%$ & $28,81 \%$ & 62 & $100 \%$ \\
\hline
\end{tabular}

$\chi^{2}=32,633 ; \mathrm{p}<0,05$.

\section{Discusión}

Uno de los resultados que más llama la atención es la poca información de la que disponen los usuarios acerca de variables relacionadas con la atención en Salud Mental. Podríamos pensar que esto se debe a que la persona nunca ha acudido a nuestros servicios, desconoce el funcionamiento de los mismos y por tanto no tiene una expectativa concreta. Sin embargo, destaca el hecho de que incluso en las variables referentes a sus preferencias un amplio porcentaje de sujetos responde no sabe/no contesta. Esto probablemente implica un amplio desconocimiento del tipo de intervenciones que se realizan, de las condiciones necesarias para llevarlas a cabo y de las ventajas e inconvenientes de cada una, lo que limita la capacidad de decisión del paciente. De ahí que un 69,4\% responda que no sabe si prefiere psicólogo clínico o psiquiatra y un $48,8 \%$ no sabe si desea tratamiento psicológico o psicofarmacológico. Además, a la vista de estos datos, el que una persona haya planteado preferir a un profesional $\mathrm{u}$ otro no garantiza que tenga la información suficiente para tomar dicha decisión.

Esto está relacionado con la información proporcionada por parte del derivante (además de con cuestiones asociadas al propio paciente que no son objeto de análisis aquí). En general comprobamos que es escasa o en cualquier caso el usuario no la recuerda. La información de alternativas de tratamiento solo se dio (según los participantes) en un $11,8 \%$ de los casos. 
Este dato concuerda con estudios realizados desde la perspectiva del derivante. En un estudio realizado en Córdoba se encontró que casi el 80\% de los MAP reconocían no utilizar el consentimiento informado sobre el tratamiento, ni explicar que existen alternativas para solucionar los problemas como el tratamiento psicológico, el tratamiento combinado u otras (21).

Este dato concuerda con nuestra experiencia clínica, dado que no son infrecuentes las derivaciones entre psicólogos clínicos y psiquiatras después de la primera consulta, debido a que el paciente no acepta el tipo de tratamiento que le plantea el clínico. Esta situación tiene consecuencias tanto clínicas como económicas.

A pesar de ello la mayoría de los usuarios se encuentran satisfechos y conformes con la derivación, y tienen confianza en la ayuda que recibirán de Salud Mental. Este es un punto de partida importante para un tratamiento exitoso, pero ajustar las expectativas mejoraría el proceso posterior, acerca del cual los usuarios parecen estar ampliamente desinformados. Por ejemplo, sería fundamental que conociesen el efecto limitado que pueden tener los fármacos en la solución de un determinado problema, el esfuerzo y cierto grado de malestar emocional que implica la psicoterapia, o hasta dónde llegan nuestras posibilidades de ayudarles con problemas de la vida cotidiana o sucesos estresantes.

En cuanto al profesional preferido, parece existir una importante desinformación respecto a las diferencias entre ellos, siendo la preferencia por el psicólogo clínico ligeramente superior. Encontramos mayores diferencias en la preferencia de tratamiento, siendo mayor el porcentaje que prefieren psicoterapia, llevada a cabo en su mayoría por psicólogos clínicos. Este dato coincide con lo que plantea la Guía de Práctica Clínica para Ansiedad en Atención Primaria (22) y con estudios previos acerca de las preferencias de los usuarios (16-17). Además, en el momento de la derivación los participantes dicen tener un elevado nivel de locus de control interno, lo que favorece este tipo de actuaciones.

Sin embargo, en la actualidad las intervenciones psicológicas en Atención Primaria son escasas o inexistentes y en la Atención Especializada los recursos son tan limitados que apenas puede ofertarse esta alternativa de tratamiento en condiciones óptimas (23). En cualquier caso, parece fundamental explorar la información que subyace a estas preferencias, con el objetivo de conocer si se trata de una decisión informada.

En general existe una coincidencia entre la preferencia de profesional y de tratamiento con el terapeuta asignado. Esto es algo importante e indicador de una buena práctica. Sin embargo, parece que son muchos los participantes que no conocen las diferencias entre los distintos tipos de intervenciones. Además, según nuestra experiencia diaria la asignación a un profesional determinado según las preferencias del paciente no se hace de forma sistemática ya que no se les pregunta directamente, por lo que puede depender más bien de la demanda del derivante o de otras variables no incluidas en esta investigación. 
En definitiva, consideramos que de este estudio se pueden derivar las siguientes conclusiones fundamentales:

- Es preciso proporcionar a los Médicos de Atención Primaria las condiciones necesarias (formación, tiempo) para que puedan informar a los pacientes acerca de lo que les ocurre y de las alternativas de tratamiento disponible. De esta forma, y siempre teniendo en cuenta el criterio clínico, el usuario estará en posesión de los conocimientos suficientes para elegir uno u otro tratamiento. Además, esto permitirá que el paciente acuda al CSM con unas expectativas más claras tanto a nivel de resultado y como de la terapia, lo que sin duda repercutirá en su implicación como agente del cambio y en la evolución posterior.

- Si bien parece que existe una coincidencia entre el profesional y tipo de terapia preferido y el profesional asignado, resulta fundamental que tras haber mejorado el proceso de toma de decisiones del paciente, se sistematice el sistema de asignación a cada tipo de profesional, teniendo en cuenta tanto preferencias como otras variables clínicas.

- A pesar de la desinformación, de forma preliminar podemos concluir que los usuarios prefieren en mayor medida tratamientos psicológicos. La aplicación de este tipo de intervenciones requiere unas condiciones determinadas (24), que no siempre se cumplen en la práctica clínica. En cualquier caso, sería interesante repetir la encuesta de preferencias tras haber informado debidamente a los usuarios.

Por último, cabe hablar de las limitaciones del estudio. Algunas de ellas son compartidas con la primera parte de la investigación, como por ejemplo la no distinción entre datos de las diferentes áreas sanitarias, la pérdida de información acerca del total de los pacientes derivados a los CSM y la no aleatorización de la muestra.

Otras limitaciones más específicas de este trabajo tienen que ver por ejemplo con la no exploración sistemática de todos los tipos de expectativas que pueden ser relevantes en el proceso de derivación. Además, en futuras investigaciones sería interesante utilizar un método de evaluación cuantitativo, dado que aportaría mayor fiabilidad y exhaustividad al proceso de recogida de información.

Una cuestión importante sería llevar a cabo un mayor trabajo de delimitación en el estudio de los conceptos de expectativas, preferencias y motivación al cambio (9), que no son equivalentes, así como determinar el peso específico de cada uno de estos aspectos en la intervención.

Por último, dados los resultados encontrados, consideramos fundamental explorar directamente la información que los sujetos tienen acerca de los distintos tipos de tratamientos y de las tareas de cada tipo de profesional. En este estudio estamos deduciendo falta de conocimiento derivada de la respuesta no sabe/no contesta ante la pregunta por sus preferencias, pero no hemos constatado directamente la información que los sujetos tienen. 
ORIGINALES Y REVISIONES

Como futura línea de investigación se propone comprobar de forma empírica si intervenir mejorando la información de los usuarios y ajustando expectativas acerca del proceso tiene un efecto positivo y de qué tipo.

\section{BIBLIOGRAFÍA}

(1) Lambert MJ, Anderson EM. Assesment for the time-limited psychotherapies. Annual Review of Psychiatry 1996; 15, 23-47.

(2) Wampold BE. The great psychotherapy debate: Models, methods, and findings (Vol. 9). Routledge, 2013.

(3) Amigo Vázquez I, Fernández Rodríguez C, Pérez Álvarez M. Placebo. En: Manual de Psicología de la Salud, pp. 153 - 159. Madrid, Pirámide, 2003.

(4) Fournier JC, DeRubeis RJ, Hollon SD, Dimidjian S, Amsterdam JD, Shelton RC, Fawcett, J. Antidepressant drug effects and depression severity: a patient-level meta-analysis. Jama 2010; 303(1), 47-53.

(5) Kirsch I, Deacon BJ, Huedo-Medina TB, Scoboria A, Moore TJ, Johnson BT. Initial severity and antidepressant benefits: a meta-analysis of data submitted to the Food and Drug Administration. PLoS medicine 2008; 5(2), e45.

(6) Ross C, Read J. Los fármacos antipsicóticos: mitos y realidades. En: Modelos de locura. Read J, Mosher L, Bentall R (eds), pp. 123 - 139. Barcelona, Herder, 2004.

(7) Ezama E, Fontanil Y, Alonso Y. Calidad de la sesión, ajuste en las tareas y efectividad de las sesiones psicoterapéuticas. International Journal of Psychology and Psychological Therapy 2012; 12(2), 245-260.

(8) Grosse Holtforth M, Wilm K, Beyermann S, Rhode A, Trost S, Steyer R. Differential change in integrative psychotherapy: A re-analysis of a change-factor based RCT in a naturalistic setting. Psychotherapy Research 2011; 21(6), 631-643.

(9) Constantino MJ, Ametrano RM, Greenberg RP. Clinician interventions and participant characteristics that foster adaptive patient expectations for psychotherapy and psychotherapeutic change. Psychotherapy 2012; 49(4), 557.

(10) Martínez O, Beitia M, Araluce K, Ayerra JM, Cela C, Grijalvo J et al. Estudio de las primeras consultas en un Centro de Salud Mental (II). Características clínicas, sucesos vitales, ajuste y apoyo social, motivación y expectativas. Rev Asoc Esp Neurpsiq. 1997; 17 (62), 203-222.

(11) Pekarik G. Psychotherapy abbreviation. A practical guide. New York: The Haworth Press, 1996.

(12) Fernández Méndez J, Luego Castro MA, García Haro J, Cabero Álvarez A, González Díaz, MD, Braña Menéndez B, Cuesta Izquierdo M, Caunedo Riesco, PJ. Efectividad de la psicoterapia breve en los centros de salud mental I: Diseño, tratamientos y procedimiento. Rev Asoc Esp Neurpsiq. 2010; 30 (108), 563-580.

(13) Fernández Méndez J, Luego Castro MA, García Haro J, Cabero Álvarez A, González Díaz, MD, Braña Menéndez B, Cuesta Izquierdo M, Caunedo Riesco, PJ.Efectividad de la psicoterapia breve en los centros de salud mental II: Resultados. Rev Asoc Esp Neurpsiq. 2011; 31 (109), 13-29.

(14) Pekarik G. Beyond effectiveness: Users of consumer-oriented criteria in defining treatment success. En: Giles TR (eds), Handbook of effective psychotherapy, pp. 409-436. New York: Plenun, 1993. 
ORIGINALES Y REVISIONES

(15) Retolaza A, Grandes G. Expectativas y satisfacción de los usuarios de un centro de salud mental. Actas Esp. Psiquiatr. 2003; 31 (4), 171-176.

(16) Pastor Sirera, J. El psicólogo en atención primaria: un debate necesario en el sistema nacional de salud. Papeles del Psicólogo. 2008; 29 (3), 271-90.

(17) OCU. Menos fármacos, más psicoterapia. Revista OCU-Salud. 2012; 100, 11-14.

(18) Feixas G, Miró MT. Aproximaciones a la psicoterapia. Barcelona, Paidós Ibérica, 1993.

(19) Norcross JC y Wampold BE. What works for whom. Tailoring psychotherapy to the person. Journal of Clinical Psychology 2011; 67, 127-132.

(20) Miller WR, Rollnick S. La entrevista motivacional. Preparar para el cambio de conductas adictivas. Barcelona, Paidós Ibérica, 1999.

(21) Moreno E, Moriana JA. El tratamiento de problemas psicológicos y de salud mental en Atención Primaria. Análisis y perspectiva desde el punto de vista de los médicos de familia. Comunicaciones de la Jornada Psicología Clínica en Atención Primaria: Una necesidad y derecho de las personas. Madrid: 19 Noviembre 2012. Disponible en http://www.cop.es/index. php?page=jornadapcap [Consulta: 19-10-2013]

(22) Unidad de Evaluación de Tecnologías Sanitarias. Agencia Laín Entralgo. Guías de Práctica Clínica en el SNS. Guía de Práctica Clínica para el Manejo de Pacientes con Trastornos de Ansiedad en Atención Primaria. Madrid, MSC 2008.

(23) Jiménez Estévez JF. El papel del Observatorio de Salud Mental y los criterios de calidad asistencial de la AEN en la evaluación y planificación asistencial. Recursos de profesionales en CSM y de dispositivos de atención a pacientes con TMG en España. Rev. Asoc. Esp. Neuropsiq. 2011, vol. 31 no.4 Madrid oct.-dic.

(24) Estrategia en Salud Mental Sistema Nacional de Salud 2009-2013. Ministerio de Sanidad, Política Social e Igualdad, 2011. Disponible en http://www.msps.es/organizacion/sns/ planCalidadSNS/docs/saludmental/SaludMental2009-2013.pdf 